\title{
BOUNDED SEQUENCE-TO-FUNCTION HAUSDORFF TRANSFORMATIONS
}

\author{
CONSTANTINE GEORGAKIS
}

(Communicated by R. Daniel Mauldin)

ABSTRACT. Let

$$
(T a)(y)=\sum_{n=0}^{\infty}(-y)^{n} \frac{g^{(n)}(y)}{n !} a_{n}, \quad y \geq 0,
$$

be the sequence-to-function Hausdorff transformation generated by the completely monotone function $g$ or, what is equivalent, the Laplace transform of a finite positive measure $\sigma$ on $[0, \infty)$. It is shown that for $1 \leq p \leq \infty, T$ is a bounded transformation of $l^{p}$ with weight $\Gamma(n+s+1) / n$ ! into $L^{p}[0, \infty)$ with weight $y^{s}, s>-1$, whose norm $\|T\|=\int_{0}^{\infty} t^{-(1+s) / p} d \sigma(t)=C(p, s)$ if and only if $C(p, s)<\infty$, and that for $1<p<\infty,\|T a\|_{p, s}<C(p, s)\|a\|_{p, s}$ unless $a_{n}$ is a null sequence. Furthermore, if $1<p<r<\infty, 0<\lambda<1$, $1 / r=1 / p+\lambda-1$ and $\sigma$ is absolutely continuous with derivatives $\psi$ such that the function $\psi_{r}(t)=t^{-1 / r} \psi(t)$ belongs to $L^{1 / \lambda}[0, \infty)$, then the transformation $\left(T_{\lambda} a\right)(y)=y^{1-\lambda}(T a)(y)$ is bounded from $l^{p}$ to $L^{r}[0, \infty)$ and has norm $\left\|T_{\lambda}\right\| \leq\left\|\psi_{r}\right\|_{1 / \lambda}$. The transformation $T$ includes in particular the Borel transform and that of generalized Abel means. These results constitute an improved analogue of a theorem of Hardy concerning the discrete Hausdorff transformation on $l^{p}$ which corresponds to a totally monotone sequence, and lead to improved forms of some inequalities of Hardy and Littlewood for power series and moment sequences.

Hardy showed that the discrete Hausdorff transformation

$$
(H a)_{n}=\sum_{k=0}^{n}\left(\begin{array}{l}
n \\
k
\end{array}\right)\left(\Delta^{n-k} \mu_{k}\right) a_{k}, \quad 1<p<\infty,
$$

where $\mu_{n}$ is a totally monotone sequence or, equivalently, $\mu_{n}=\int_{0}^{1} t^{n} d \sigma(t)$, where $\sigma$ is a probability measure on $[0,1]$, such that $\sigma(\{0\})=0$ and $C_{p}=\int_{0}^{1} t^{-1 / p} d \sigma(t)<$ $\infty$, is a bounded transformation on $l^{p}$ and $\|H a\|_{p} \leq C_{p}\|a\|_{p}$. Moreover, $C_{p}$ is the best possible constant and exact equality occurs only if $a_{n}$ is a null sequence or $T$ is the identity transformation [2] or [3].

Our aim here is to establish the analogue of Hardy's Theorem for the Hausdorff transformation generated by a completely monotone function and to strengthen it in the case of the measure $\sigma$ is an absolutely continuous with derivative in $L^{p}$. Then we show how these results lead to certain $L^{p}$ inequalities for the Borel transform and the transformation of generalized Abel means. These in turn give rise to improved

Received by the editors April 1, 1987.

1980 Mathematics Subject Classification (1985 Revision). Primary 26D15; Secondary 42G10.

Key words and phrases. Hausdorff, transformation, bounded, Abel means, Borel transform, completely monotone, power series, moments. 
versions of some inequalities for power series and moment sequences of Hardy and Littlewood [4].

The Hausdorff transformation generated by a completely monotone function $g$ or, what is equivalent (see [11]), the Laplace transform of a finite positive Borel measure $\sigma$ on $[0, \infty)$ is given by

$$
T a(y)=\sum_{n=0}^{\infty}(-y)^{n} \frac{g^{(n)}(y)}{n !} a_{n}=\sum_{n=0}^{\infty} a_{n} \int_{0}^{\infty} e^{-y t} \frac{(y t)^{n}}{n !} d \sigma(t), \quad y \geq 0 .
$$

The transformation $T$ which maps a sequence $a_{n}$ to the function $T a(y), y \geq 0$, is known as the Henriksson transformation or $J$-transform. It reduces to Borel summation when $\sigma$ is a unit mass at 1 and to the generalized Abel means of order $\nu>0$ when $\sigma$ is the gamma probability density $t^{\nu-1} e^{-t} / \Gamma(\nu)$. The transformation $T$ was introduced by Henriksson [5] as a generalization of Abel and Borel summability, and its summability properties have been studied by Henriksson [6], Jakimovski [7], Leviatan and Lorch [9], Borwein [1], Renyi [10] and others. Theorems 1 and 2 below describe some of the $L^{p}$-continuity properties of the transformation $T$.

In the sequel we denote by $l^{p, s}$ and $L^{p, s}[0, \infty)$ the space of sequences $a=\left\{a_{n}\right\}_{n=0}^{\infty}$ and functions, $f(y), y \geq 0$, respectively, such that

$$
\begin{aligned}
& \|a\|_{p, s}^{p}=\sum_{n=0}^{\infty}\left|a_{n}\right|^{p}(n+1)_{s}<\infty, \\
& \|f\|_{p, s}^{p}=\int_{0}^{\infty}|f(y)|^{p} y^{s} d y<\infty,
\end{aligned}
$$

where $s>-1,(n+1)_{s}=\Gamma(n+s+1) / n$ ! is the Pochhamer symbol, and $p^{\prime}=p /(p-1)$ is the conjugate index of $p$. We shall also need the following inequalities for the gamma function and the binomial coefficients.

LEMMA 1. (a) If $u>0, v>0$ then,

$$
\begin{aligned}
\Gamma(u+v) / \Gamma(u) & \leq u^{v} \quad \text { for } 0<v \leq 1, \text { and } \\
& \geq u^{v} \quad \text { for } v>1,
\end{aligned}
$$

(b) $(n+1)_{s} \leq(n+1)^{s+1} /(n+s+1)$ for $n \geq 0,-1<s<0$,

(c) $(n+1)_{s} \leq(n+s+1)^{s}$ for $n \geq 0, s \geq 0$.

PROOF. (a) is simply a consequence of Jensen's inequality;

$$
\left[\frac{\Gamma(u+v)}{\Gamma(u)}\right]^{1 / v}=\left[\int_{0}^{\infty} \frac{t^{v} t^{u-1} e^{-t} d t}{\Gamma(u)}\right]^{1 / v} \leq \text { or } \geq \int_{0}^{\infty} \frac{t^{u} e^{-t} d t}{\Gamma(u)}=u
$$

depending on $v \leq 1$ or $v>1$. To prove (b) let $u=n+1, v=s+1$ in (a). Then $u>0,0<v \leq 1$, and

$$
(n+1)_{s}=\frac{\Gamma(n+s+2)}{\Gamma(n+1)(n+s+1)} \leq \frac{(n+1)^{s+1}}{n+s+1} .
$$

Finally, to prove (c) let $u=n+[s+1], v=s+1-[s+1]$ in (a). Then $u>0$, $0<v<1$ since we may assume $s>0$, and by (a) we have

$$
\begin{aligned}
n !(n+1)_{s}(n+[s+1]) & =(n+[s+1]) ! \Gamma(n+[s+1]+v) / \Gamma(n+[s+1]) \\
& \leq n !(n+[s+1])^{[s+1]}(n+[s+1])^{v} .
\end{aligned}
$$


Hence,

$$
(n+1)_{s} \leq(n+[s+1])^{s} \leq(n+s+1)^{s} .
$$

LEMMA 2. If $C_{n \nu}=\left(\begin{array}{c}n+\nu-1 \\ \nu-1\end{array}\right), n$ is an integer and $\nu$ is real, then we have

(a) $C_{n \nu} \geq(n+1)^{\nu-1} / \max (\Gamma(\nu), 1)$ for $n \geq 0, \nu>0$,

(b) $C_{n \nu} \leq(n+1)^{\nu-1} / \min (\Gamma(\nu), 1)$ for $n \geq 0, \nu>1$,

(c) $C_{n \nu} \leq n^{\nu-1} / \Gamma(\nu)$ for $n \geq 1,0<\nu \leq 1$.

PROOF. In proving (a) and (b) we may assume $n \geq 1$, since both are clearly valid for $n=0$. For $0<\nu<1$, (a) is equivalent to $\Gamma(n+1) / \Gamma(n+\nu) \leq(n+1)^{1-\nu}$ which follows by Lemma 1(a) with $u=n+\nu, v=1-\nu$. For $\nu>2$, (a) also follows from Lemma 1(a) with $u=n+1, v=\nu-1$. To prove (a) for $1<\nu<2$, we have

$$
\begin{aligned}
{\left[\frac{\Gamma(n+1) \Gamma(\nu)}{\Gamma(n+\nu)}\right]^{1 /(\nu-1)} } & =\left[\int_{0}^{1}(1-x)^{\nu-1} x^{n-1} n d x\right]^{1 /(\nu-1)} \\
& \leq \int_{0}^{1}(1-x) x^{n-1} n d x=\frac{1}{n+1}, \quad \text { since } \frac{1}{\nu-1}>1 .
\end{aligned}
$$

The last inequality gets reversed when $1 /(\nu-1)<1$, and establishes (b) for $\nu>2$. For $1<\nu<2$, (b) is equivalent to $\Gamma(n+1+(\nu-1)) / \Gamma(n+1) \leq(n+1)^{\nu-1}$ which is Lemma 1(a) with $u=n+1, v=\nu-1$. Finally, for $0<\nu \leq 1$, (c) follows similarly from the inequality $\Gamma(n+\nu) / n \Gamma(n) \leq n^{\nu-1}$ which is Lemma 1 (a) with $u=n, v=\nu$.

THEOREM 1. Let $\sigma$ be a complex Borel measure of finite variation on $[0, \infty)$ such that $\sigma(\{0\})=0$ and $T$ be the transformation given by

$$
(T a)(y)=\sum_{n=0}^{\infty} a_{n} \int_{0}^{\infty} e^{-y t} \frac{(y t)^{n}}{n !} d \sigma(t), \quad y \geq 0
$$

where

$$
C(p, s)=\int_{0}^{\infty} t^{-(1+s) / p} d|\sigma|(t)<\infty, \quad s>-1,
$$

and $a=\left\{a_{n}\right\}_{n=0}^{\infty}$ is a sequence in $l^{p, s}$. Then we have

(a) $T$ is a bounded transformation of $l^{p, s}$ into $L^{p, s}[0, \infty)$ and

$$
\|T a\|_{p, s} \leq C(p, s)\|a\|_{p, s}, \text { i.e., }\|T\| \leq C(p, s) \text { for } 1 \leq p \leq \infty .
$$

(b) If in addition $\sigma$ is a positive measure, then (i) $\|T a\|_{p, s}<C(p, s)\|a\|_{p, s}$ for $1<p<\infty$ unless the sequence $a_{n}$ is null; (ii) $\|T\|=C(p, s)$ for $1 \leq p \leq \infty$; (iii) the condition $C(p, s)<\infty$ is also necessary for $T$ to be bounded.

ProOF. For $p=\infty$, (a) is clearly true, and for $p=1$, it follows by Fubini's theorem. Let $1<p<\infty, a \in l^{p, s}$, and consider the transformation $E_{t}, t>0$, given by

$$
\left(E_{t} a\right)(y)=\sum_{n=0}^{\infty} e^{-y t} \frac{(y t)^{n}}{n !}\left|a_{n}\right|, \quad y \geq 0 .
$$


Then

$$
\begin{aligned}
\left(E_{t} a\right)(y) & \left.=\sum_{n=0}^{\infty}\left(e^{-y t} \frac{(y t)^{n}}{n !}\left|a_{n}\right|\right)^{p}\right)^{1 / p}\left(e^{-y t} \frac{(y t)^{n}}{n !}\right)^{1 / p^{\prime}} \\
& \leq\left(\sum_{n=0}^{\infty} e^{-y t} \frac{(y t)^{n}}{n !}\left|a_{n}\right|^{p}\right)^{1 / p}
\end{aligned}
$$

by Hoelder's inequality. Moreover, if $t>0, y>0$ and we have exact equality, then

$$
\left|a_{n}\right|^{p} e^{-y t}(y t)^{n} / n !=K e^{-y t} \frac{(y t)^{n}}{n !} \text { for } n \geq 0
$$

for some positive constant $K$. This implies that $\left|a_{n}\right|^{p}=K$ for $n \geq 0$ and that $\|a\|_{p, s}^{p}=K^{p} \sum_{n=0}^{\infty}(n+1)_{s}$, which is divergent for $s>-1$ and leads to contradiction. Therefore,

$$
\left(E_{t} a\right)(y)^{p}<\sum_{n=0}^{\infty} e^{-y t} \frac{(y t)^{n}}{n !}\left|a_{n}\right|^{p}
$$

unless the sequence $a_{n}$ is null. Let $f \in L^{p^{\prime}, s}[0, \infty)$. Using the Hoelder's inequality for a double integral and the preceding inequality for $\left(E_{t} a\right)(y)^{p}$, we get

$$
\begin{aligned}
|(T a, f)|=\left|\int_{0}^{\infty}(T a)(y) f(y) y^{s} d y\right| \\
\quad \leq \int_{0}^{\infty}|f(y)| y^{s} d y \int_{0}^{\infty}\left(E_{t} a\right)(y) d|\sigma|(t) \\
\quad \leq \int_{0}^{\infty} \int_{0}^{\infty}\left[\left(E_{t} a\right)(y) t^{(1+s) / p p^{\prime}}\right]\left[|f(y)| t^{-(1+s) / p p^{\prime}}\right] y^{s} d y d|\sigma|(t) \\
\quad \leq\left[\int_{0}^{\infty} \int_{0}^{\infty}\left(E_{t} a\right)(y)^{p} t^{(1+s) / p^{\prime}} y^{s} d y d|\sigma|(t)\right]^{1 / p} C(p, s)^{1 / p^{\prime}}\|f\| p^{\prime}, s \\
\quad \leq\left[\int_{0}^{\infty} \int_{0}^{\infty}\left(\sum_{n=0}^{\infty} e^{-y t} \frac{(y t)^{n}}{n !}\left|a_{n}\right|^{p}\right) t^{(1+s) / p^{\prime}} y^{s} d y d|\sigma|(t)\right]^{1 / p} C(p, s)^{1 / p^{\prime}}\|f\|_{p^{\prime}, s} \\
\quad=C(p, s)^{1 / p}\|a\|_{p, s} C(p, s)^{1 / p^{\prime}}\|f\|_{p^{\prime}, s} \\
\quad=C(p, s)\|a\|_{p, s}\|f\|_{p^{\prime}, s .}
\end{aligned}
$$

Hence, $\|T a\|_{p, s} \leq C(p, s)\|a\|_{p, s}$. Furthermore, the preceding argument shows that if $\sigma$ is positive, $a_{n} \geq 0, f \geq 0$, and $(T a, f)=C(p, s)\|a\|_{p, s}\|f\|_{p^{\prime}, s}$ then (*) holds a.e. for $y>0$ and a.e. relative to the measure $\sigma$ for $t>0$ and, hence, $a_{n}$ is a null sequence.

(b) For the rest of the proof we let $\sigma$ be a finite positive measure.

(i) Suppose $\|T a\|_{p, s}=C(p, s)\|a\|_{p, s}$ for some sequence $a_{n}$ in $l^{p, s}$. Then $a_{n} \geq 0$, because $\|T a\|_{p, s} \leq\|T|a|\|_{p, s}$, where $|a|=\left\{\left|a_{n}\right|\right\}_{n=0}^{\infty}$, and $\|T a\|_{p, s} \leq C(p, s)\|a\|_{p, s}$ by (a). Furthermore, there is $f \in L^{p^{\prime}, s}[0, \infty)$ such that

$$
|(T a, f)|=C(p, s)\|a\|_{p, s}\|f\|_{p^{\prime}, s} .
$$


Since $|(T a, f)| \leq(T a,|f|)$ for $a_{n} \geq 0$, and $|(T a, f)| \leq C(p, s)\|a\|_{p, s}\|f\|_{p^{\prime}, s}$ by (a) it follows also that $f \geq 0$. Thus $(* *)$ and, therefore, $a_{n}$ is a null sequence by the remark made at the end of the proof for (a).

(ii) We now proceed to establish that $\|T\|=C(p, s)$ or, what is equivalent, $C(p, s)$ is best possible constant for the inequality, $\|T a\|_{p, s} \leq C(p, s)\|a\|_{p, s}$ for $1 \leq p \leq \infty$. Since $\|T a\|_{p, s}=C(p, s)\|a\|_{p, s}$ for $p=1, a_{n} \geq 0$, and, for $p=\infty$ when $a_{n} \geq 0$ is a constant, we may assume $1<p<\infty$. For this it suffices to show that for each $0<\eta<1$ there exists a sequence $a_{n}$ in $l^{p, s}$ such that

$$
\|T a\|_{p, s}^{p}>(1-\eta)^{2 p+1} C(p, s)\|a\|_{p, s}
$$

Let $a_{n}=(n+s+1)^{-1 / p-\varepsilon}(n+1)_{s}^{-1 / p}$, for $\varepsilon>0, s>-1$. Then

$$
\|a\|_{p, s}^{p}=\sum_{n=0}^{\infty}(n+s+1)^{-1-\varepsilon p}
$$

and from the estimates for $(n+1)_{s}$ in Lemma 1 we obtain

$$
\begin{gathered}
a_{n} \geq(n+s+1)^{-\varepsilon}(n+1)^{-(1+s) / p} \geq(n+1)^{-(1+s) / p-\varepsilon} \text { for }-1<s<0 \\
a_{n} \geq(n+s+1)^{-(1+s) / p-\varepsilon} \text { for } s \geq 0
\end{gathered}
$$

Therefore, with $s^{*}=\max (s, 0), \gamma=1 / \Gamma((1+s) / p+\varepsilon)$ for all $\varepsilon>0, s>-1$ and positive integer $N \geq 2$, we have

$$
\begin{aligned}
& a_{n} \geq\left(n+s^{*}+1\right)^{-(1+s) / p-\varepsilon}=\gamma \int_{0}^{\infty} x^{(1+s) / p+\varepsilon-1} e^{-\left(n+s^{*}+1\right) x} d x \\
& (T a)(y) \geq \gamma \int_{0}^{\infty} d \sigma(t) \int_{0}^{\infty} x^{(1+s) / p+\varepsilon-1}\left[\sum_{n=0}^{\infty} e^{-y t} \frac{(y t)^{n}}{n !} e^{-\left(n+s^{*}+1\right) x}\right] d x \\
& =\gamma \int_{0}^{\infty} d \sigma(t) \int_{0}^{\infty} x^{(1+s) / p+\varepsilon-1} e^{-\left(s^{*}+1\right) x} e^{y t\left(e^{-x}-1\right)} d x \\
& >\gamma \int_{0}^{\infty} d \sigma(t) \int_{0}^{\infty} x^{(1+s) / p+\varepsilon-1} e^{-\left(1+s^{*}+y t\right) x} d x \\
& \text { (since } e^{-x}-1>-x \text { for } x>0 \text { ) } \\
& =\int_{0}^{\infty}\left(1+s^{*}+y t\right)^{-(1+s) / p-\varepsilon} d \sigma(t) \\
& \|T a\|_{p, s}^{p}>\int_{0}^{\infty} y^{-(1+\varepsilon p)} d y\left[\int_{0}^{\infty}\left(\frac{1+s^{*}}{y}+t\right)^{-(1+s) / p-\varepsilon} d \sigma(t)\right]^{p} \\
& >\int_{(N+s)}^{\infty} y^{-(1+\varepsilon p)}\left[\int_{1 /(N+s)}^{\infty}\left(\frac{1+s^{*}}{N+s}+t\right)^{-(1+s) / p-\varepsilon} d \sigma(t)\right]^{p} \\
& >\sum_{n=N}^{\infty}(n+s+1)^{-(1+\varepsilon p)}\left[\int_{1 /(N+s)}^{\infty}\left(\frac{1+s^{*}}{N+s}+t\right)^{-(1+s) / p-\varepsilon} d \sigma(t)\right]^{p} \text {. }
\end{aligned}
$$


Now for a given $0<\eta<1$, we choose $N \geq 2, \varepsilon_{1}>0$, and $\varepsilon_{2}>0$ in that order so that

$$
\begin{aligned}
& \int_{1 /(N+s)}^{\infty}\left(\frac{1+s^{*}}{N+s}+t\right)^{-(1+s) / p} d \sigma(t) \\
& \quad>(1-\eta) \int_{0}^{\infty} t^{-(1+s) / p} d \sigma(t)=(1-\eta) C(p, s), \\
& \int_{1 /(N+s)}^{\infty}\left(\frac{1+s^{*}}{N+s}+t\right)^{-(1+s) / p-\varepsilon} d \sigma(t) \\
& \quad>(1-\eta) \int_{1 /(N+s)}^{\infty}\left(\frac{1+s^{*}}{N+s}+t\right)^{-(1+s) / p} d \sigma(t) \text { for } 0<\varepsilon<\varepsilon_{1}, \\
& \sum_{n=N}^{\infty}(n+s+1)^{-(1+\varepsilon p)}>(1-\eta) \sum_{n=0}^{\infty}(n+s+1)^{-(1+\varepsilon p)} \text { for } 0<\varepsilon<\varepsilon_{2} .
\end{aligned}
$$

The selection of $\varepsilon_{1}$ is possible by dominated convergence since

$$
\left(\frac{1+s^{*}}{N+s}+t\right)^{-(1+s) / p-\varepsilon} \leq\left(\frac{N+s^{*}}{N+s}\right)^{\varepsilon} t^{-(1+s) / p} \quad \text { for } t \geq \frac{1}{N+s} .
$$

The selection of $\varepsilon_{2}$ is also possible in view of the equation

$$
\sum_{n=0}^{\infty}(n+s+1)^{-(1+\varepsilon p)}=\sum_{n=0}^{N-1}(n+s+1)^{-(1+\varepsilon p)}+\sum_{n=N}^{\infty}(n+s+1)^{-(1+\varepsilon p)}
$$

where the ratio of the second term on the right-hand of the above equation to that on the left-hand side increases to 1 as $\varepsilon$ decreases to zero. Thus if $\varepsilon$ is chosen smaller than both $\varepsilon_{1}$ and $\varepsilon_{2}$ and the estimates are combined we obtain

$$
\|T a\|_{p, s}^{p}>(1-\eta)^{2 p+1}\|a\|_{p, s}^{p} .
$$

(iii) Suppose $T$ is bounded and consider the transformation $T_{\delta}$ generated by the restriction of the measure $\sigma$ on $[\delta, \infty)$ for $\delta>0$. Clearly, by (a) $T_{\delta}$ is bounded and, by (ii) of (b), $\left\|T_{\delta}\right\|=\int_{\delta}^{\infty} t^{-(1+s) / p} d \sigma(t)<\infty$. Furthermore, $\left|\left(T_{\delta} a\right)(y)\right| \leq$ $\left(T_{\delta}|a|\right)(y) \leq(T|a|)(y)$ for $y \geq 0$. Hence, $\left\|T_{\delta}\right\| \leq\|T\|$ which yields that $C(p, s) \leq$ $\|T\|<\infty$ on letting $\delta \rightarrow 0$. This completes the proof of Theorem 1 .

THEOREM 2. Consider the transformation $T_{\lambda}$ given by

$$
\left(T_{\lambda} a\right)(y)=y^{1-\lambda} \sum_{n=0}^{\infty} a_{n} \int_{0}^{\infty} e^{-y t} \frac{(y t)^{n}}{n !} \psi(t) d t
$$

where

$$
C(\lambda, p, r)=\int_{0}^{\infty} t^{-1 / \lambda r}|\psi(t)|^{1 / \lambda} d t<\infty, \quad 1 / r=1 / p+\lambda-1
$$

Then $T_{\lambda}$ is a bounded transformation from $l^{p}$ to $L^{r}[0, \infty)$ for $1<p<r<\infty$, and $\left\|T_{\lambda} a\right\|_{r} \leq C(\lambda, p, r)^{\lambda}\|a\|_{p}$. Moreover, if $\psi \geq 0$, then $\left\|T_{\lambda} a\right\|_{r}<C(\lambda, p, r)^{\lambda}\|a\|_{p}$ unless $a_{n}$ is a null sequence.

ProOF. Let $f \in L^{r^{\prime}}[0, \infty), 0<\lambda<1,1<p<r<\infty$, and let $\left(E_{t} a\right)(y)$ be the transformation used in the proof of Theorem 1(a). Then an application of Hoelder's 
inequality for the indices $1 / r+1 / p^{\prime}+(1-\lambda)=1$, yields

$$
\begin{aligned}
&\left|\int_{0}^{\infty}\left(T_{\lambda} a\right)(y) f(y) d y\right| \leq \int_{0}^{\infty} \int_{0}^{\infty}\left[\left(E_{t} a\right)(y)^{p} t^{1 / \lambda p^{\prime}}|\psi(t)|^{1 / \lambda}\right]^{1 / r} \\
&\left.\cdot\left[|f(y)|^{r^{\prime}} t^{-1 / \lambda r}|\psi(t)|^{1 / \lambda}\right]^{1 / p^{\prime}}\left[E_{t} a\right)(y)^{p}|f(y)|^{r^{\prime}} y\right]^{1-\lambda} d y d t \\
& \leq {\left[\int_{0}^{\infty} \int_{0}^{\infty}\left(E_{t} a\right)(y)^{p} t^{1 / \lambda p^{\prime}}|\psi(t)|^{1 / \lambda} d y d t\right]^{1 / r} } \\
& \cdot\left[\int_{0}^{\infty} \int_{0}^{\infty}|f(y)|^{r^{\prime}} t^{-1 / \lambda r}|\psi(t)|^{1 / \lambda} d y d t\right]^{1 / p^{\prime}} \\
& \cdot\left[\int_{0}^{\infty} \int_{0}^{\infty}\left(E_{t} a\right)(y)^{p}|f(y)|^{r^{\prime}} y d y d t\right]^{1-\lambda}=P^{1 / r} Q^{1 / p^{\prime}} R^{1-\lambda}
\end{aligned}
$$

Since $1 / \lambda p^{\prime}+1 / \lambda r=1$ and

$$
\left(E_{t} a\right)(y)^{p} \leq \sum_{n=0}^{\infty} e^{-y t} \frac{(y t)^{n}}{n !}\left|a_{n}\right|^{p}
$$

by Hoelder's inequality, we have

$$
\begin{aligned}
& P \leq \int_{0}^{\infty} t^{-1 / \lambda r}|\psi(t)|^{1 / \lambda} d t\left[\sum_{n=0}^{\infty}\left|a_{n}\right|^{p} \int_{0}^{\infty} e^{-y t} \frac{(y t)^{n}}{n !} t d y\right]=C(\lambda, p, r)\|a\|_{p}^{p} \\
& Q=C(\lambda, p, r)\|f\|_{r^{\prime}}^{r^{\prime}}, \\
& R \leq \int_{0}^{\infty}|f(y)|^{r^{\prime}} d y\left[\sum_{n=0}^{\infty}\left|a_{n}\right|^{p} \int_{0}^{\infty} e^{-y t} \frac{(y t)^{n}}{n !} y d t\right]=\|f\|_{r^{\prime}}^{r^{\prime}}\|a\|_{p}^{p}
\end{aligned}
$$

Therefore,

$$
\begin{aligned}
\left|\int_{0}^{\infty}\left(T_{\lambda} a\right)(y) f(y) d y\right| & \leq C(\lambda, p, r)^{1 / r+1 / p^{\prime}}\|a\|_{p}^{p(1 / r+1-\lambda)}\|f\|_{r^{\prime}}^{r^{\prime}\left(1 / p^{\prime}+1-\lambda\right)} \\
& =C(\lambda, p, r)^{\lambda}\|a\|_{p}\|f\|_{r^{\prime}}
\end{aligned}
$$

and

$$
\left\|T_{\lambda} a\right\|_{r} \leq C(\lambda, p, r)^{\lambda}\|a\|_{p}
$$

The proof for the assertion when $\psi \geq 0$ is similar to that given for Theorem 1 .

Corollary 1 is simply the special case of Theorem 1 where the measure $\sigma$ is the unit mass at 1 , and for $s=0$ it includes an inequality of Knopp for the Borel transform of a sequence [8, Satz III].

COROllary 1. For $s>-1,1 \leq p \leq \infty$,

$$
\int_{0}^{\infty}\left|\sum_{n=0}^{\infty} e^{-y} \frac{y^{n}}{n !} a_{n}\right|^{p} y^{s} d y \leq \sum_{n=0}^{\infty}\left|a_{n}\right|^{p}(n+1)_{s}
$$

where the constant is best possible, and for $1<p<\infty$ the strict inequality is valid unless the sequence $a_{n}$ is null. 
The generalized Abel means $A^{(\nu)}(x)$ of order $\nu>0$ of a sequence $a_{n}$ and the modified moments $A_{n}^{(\nu)}, \nu>0$, of a function $a(x), 0 \leq x \leq 1$, are defined by

$$
\begin{aligned}
A^{(\nu)}(x) & =(1-x)^{\nu} \sum_{n=0}^{\infty}\left(\begin{array}{c}
n+\nu-1 \\
\nu-1
\end{array}\right) a_{n} x^{n}, \\
A_{n}^{(\nu)} & =\left(\begin{array}{c}
n+\nu-1 \\
\nu-1
\end{array}\right) \int_{0}^{1} x^{n} a(x) d x,
\end{aligned}
$$

for $0 \leq x \leq 1$, and $n \geq 0$, respectively. Note that for $\nu=1$ these are simply the ordinary Abel means and moments. In the sequel we also use the notation $A(x)$ for the power series $A^{(1)}(x) /(1-x)$ with coefficients $a_{n}$ and $A_{n}$ for the moments $A_{n}^{(1)}$ of the function $a(x)$.

COROLlaRY 2. For $1<p<\infty, \nu>0$, and $-1<s<\nu p-1$,

(a) $\int_{0}^{1}\left|A^{(\nu)}(x)\right|^{p}(1-x)^{-s-2} x^{s} d x<\left[\frac{\Gamma((\nu p-s-1) / p)}{\Gamma(\nu)}\right]^{p} \sum_{n=0}^{\infty}\left|a_{n}\right|^{p}(n+1)_{s}$,

(b)

$$
\begin{aligned}
\sum_{n=0}^{\infty} \mid & \left.A_{n}^{(\nu)}\right|^{p}(n+1)_{s}^{1-p} \\
& <\left[\frac{\Gamma\left(\left(\nu p^{\prime}-s-1\right) / p^{\prime}\right)}{\Gamma(\nu)}\right]^{p} \int_{0}^{1}|a(x)|^{p}(1-x)^{(2-\nu) p-s-2} x^{s} d x
\end{aligned}
$$

unless the sequence $a_{n}$ is null or $a(x)$ is null a.e., and the constants are best possible.

PROOF. (a) follows from Theorem 1 on putting the gamma probability density with parameter $\nu>0$ for the measure $\sigma$. Then

$$
\begin{aligned}
C(p, s) & =\frac{\Gamma((\nu p-s-1) / p)}{\Gamma(\nu)} \text { for }-1<s<\nu p-1, \\
(T a)(y) & =\sum_{n=0}^{\infty} a_{n} \int_{0}^{\infty} \frac{e^{-y t} \frac{(y t)^{n}}{n !} t^{\nu-1} e^{-t} d t}{\Gamma(\nu)} \\
& =(1+y)^{-\nu} \sum_{n=0}^{\infty}\left(\begin{array}{c}
n+\nu-1 \\
\nu-1
\end{array}\right) a_{n}\left(\frac{y}{1+y}\right)^{n},
\end{aligned}
$$

and, the change of variable $x=y /(1+y)$, which maps $[0, \infty)$ onto $[0,1)$ so that $(T a)(y)=A^{(\nu)}(x)$ and $y^{s} d y=(1-x)^{-s-2} x^{s} d x$, shows that (a) is simply a restatement of (a) and (b) of Theorem 1. (b) Let $\sigma$ be a finite positive measure such that $C\left(p^{\prime}, s\right)<\infty$. Then it is easy to see that the transformation $T^{*}$ given for $f \in L^{p, s}[0, \infty)$ by

$$
\left(T^{*} f\right)_{n}=\int_{0}^{\infty} f(y) y^{s} d y \int_{0}^{\infty} \frac{e^{-y t}(y t)^{n} d \sigma(t)}{\Gamma(n+s+1)}
$$

is the adjoint of the transformation $T$ in Theorem 1. Hence, $T^{*}$ is a bounded transformation from $L^{p, s}[0, \infty)$ into $l^{p, s},\left\|T^{*}\right\|=\|T\|=C\left(p^{\prime}, s\right)$ and $\left\|T^{*} f\right\|_{p, s}<$ $C\left(p^{\prime}, s\right)\|f\|_{p, s}$ unless $f$ is null a.e. Let $\sigma$ be the gamma probability density as before in (a). Then

$$
C\left(p^{\prime}, s\right)=\Gamma\left(\left(\nu p^{\prime}-s-1\right) / p^{\prime}\right) / \Gamma(\nu)
$$


and

$$
\left(T^{*} f\right)_{n}=(n+1)_{s}^{-1}\left(\begin{array}{c}
n+\nu-1 \\
\nu-1
\end{array}\right) \int_{0}^{\infty}\left(\frac{y}{1+y}\right)^{n}(1+y)^{-\nu} f(y) y^{s} d y
$$

Now let $A_{n}^{(\nu)}$ be the modified moments of the function $a(x), 0 \leq x \leq 1$. Then, through the change of variable $y=x /(1-x)$ which maps $[0,1)$ onto $[0, \infty)$ so that $d x=d y /(1+y)^{2}$, and $y^{s} d y=(1-x)^{-s-2} x^{s} d x$, we see that $A_{n}^{(\nu)} /(n+1)_{s}=\left(T^{*} f\right)_{n}$ where $f(y)=(1+y)^{\nu-2} a(y /(1+y))$, and conclude that the assertion made earlier concerning the transformation $T^{*}$ translates precisely into that of (b) which we wanted to show.

COROLlaRY 3. If $1<p<r<\infty, 1 / r=1 / p+\lambda-1, \nu>1 / p$, then

$$
\begin{aligned}
& {\left[\int_{0}^{1}\left|A^{(\nu)}(x)\right|^{r}(1-x)^{-r / p-1} x^{r / p-1} d x\right]^{1 / r}} \\
& \quad<\left[\frac{\lambda^{(\nu p-1) / \lambda p} \Gamma((\nu p-1) / \lambda p)}{\Gamma(\nu)}\right]^{\lambda}\left[\sum_{n=0}^{\infty}\left|a_{n}\right|^{p}\right]^{1 / p}
\end{aligned}
$$

unless the sequence $a_{n}$ is null.

The proof for this is identical with that given for Corollary 2(a) except that here Theorem 2 is used in place of Theorem 1.

REMARK. Theorems 1,2 , and Corollaries 2(a), 3 remain valid when $\|a\|_{p}$ is replaced by $\|B a\|_{p}$ where $B a$ is the Borel transform of the sequence $a_{n}$. The proofs are similar except for the assertions about the constants being best possible, which follow by Corollary 1 from Theorem 1(b) and Corollary 2(a).

Hardy and Littlewood in [4] developed certain weighted normed inequalities, which relate the norm of a power series $A(x)$ to that of its coefficients $a_{n}$, and the norm of a moment sequence $A_{n}$ to that of its corresponding function $a(x)$, under the assumption that $a_{0}=0$ and with the exclusion of the term $A_{0}$ from their estimates. We now want to show how the inequalities obtained for the generalized Abel means $A^{(\nu)}(x)$ and the modified moments $A_{n}^{(\nu)}$ lead to improved forms of their inequalities so that no restriction is made on $a_{0}$ and the term $A_{0}$ is also included. Theorem 3 corresponds to Theorems 3 and 4 of Hardy and Littlewood which are the most inclusive results for $p>1$ in [4]. The inequalities listed below constitute a considerable improvement of the inequalities $\left(A_{0}\right),\left(B_{0}\right),\left(C_{0}\right)$ and $\left(D_{0}\right)$ in $[4] .\left(A_{0}\right)$ and $\left(\mathrm{D}_{0}\right)$ are simply special cases of Corollary 2 where $\nu=1, s=0$. Similarly, $\left(\mathrm{B}_{0}\right)$ and $\left(\mathrm{C}_{0}\right)$ are special cases of Theorem 3 where $r=p, q=p$ in (a), and $r=p, q=p^{\prime}$ in (b). Note that the constants in $\left(\mathrm{B}_{0}\right)$ for $2 \leq p<\infty$ and in $\left(\mathrm{C}_{0}\right)$ for $1<p \leq 2$ are, respectively, $\Gamma(1 / p)$ and $\Gamma\left(1 / p^{\prime}\right)$. But $\left(\mathrm{A}_{0}\right)$ or, what is equivalent, Corollary $1(\mathrm{a})$ is false for $p=1$. Because Theorem 1 is false in the case of the ordinary Abel means, i.e., when $\nu=1, p=1, s=0$, and the constant $C(p, s)$ is infinite. $\left(\mathrm{C}_{0}\right)$ is also false for $p=1$, since $A_{n}=1 / \log (n+2), n \geq 0$, is a moment sequence and the series in $\left(\mathrm{C}_{0}\right)$ is divergent $[3, \S 11.13]$. However, $\left(\mathrm{A}_{0}^{*}\right)$ and $\left(\mathrm{C}_{0}^{*}\right)$, which are analogues of Carleman's inequality, serve as substitutes for $\left(\mathrm{A}_{0}\right)$ and $\left(\mathrm{C}_{0}\right)$ when $p=1, a(x) \geq 0$, $a_{n} \geq 0$, and where $\gamma$ is Euler's constant [5, p. 249]. They are derived by replacing 
$a_{n}$ by $a_{n}^{1 / p}$ in $\left(\mathrm{A}_{0}\right), a(x)$ by $a(x)^{1 / p}$ in $\left(\mathrm{C}_{0}\right)$ and then taking the limit as $p \rightarrow \infty$.

$$
\int_{0}^{1}|A(x)|^{p}(1-x)^{p-2} d x<\Gamma\left(\frac{1}{p^{\prime}}\right)^{p} \sum_{n=0}^{\infty}\left|a_{n}\right|^{p}
$$

$$
\int_{0}^{1}|A(x)|^{p} d x<\left[\Gamma\left(\frac{1}{p}\right) \max \left(\frac{1}{\Gamma(2 / p)}, 1\right)\right]^{p} \sum_{n=0}^{\infty}\left|a_{n}\right|^{p}(n+1)^{p-2}
$$

$$
\sum_{n=0}^{\infty}\left|A_{n}\right|^{p}(n+1)^{p-2}<\left[\Gamma\left(\frac{1}{p^{\prime}}\right) \max \left(\frac{1}{\Gamma\left(2 / p^{\prime}\right)}, 1\right)\right]^{p} \int_{0}^{1}|a(x)|^{p} d x
$$

$$
\sum_{n=0}^{\infty}\left|A_{n}\right|^{p}<\Gamma\left(\frac{1}{p}\right)^{p} \int_{0}^{1}|a(x)|^{p}(1-x)^{p-2} d x
$$

unless the sequence $a_{n}$ is null or $a(x)$ is null a.e., respectively, for $1<p<\infty$, and the constants are best possible in $\left(\mathrm{A}_{0}\right)$ and $\left(\mathrm{D}_{0}\right)$ for $1<p<\infty$, in $\left(\mathrm{B}_{0}\right)$ for $2 \leq p<\infty$, and in $\left(\mathrm{C}_{0}\right)$ for $1<p \leq 2$.

$$
\begin{aligned}
& \int_{0}^{1}(1-x)^{-2} \exp \left[(1-x) \sum_{n=0}^{\infty}\left(\log a_{n}\right) x^{n}\right] d x \\
& \leq e^{\gamma} \sum_{n=0}^{\infty} a_{n}, \quad a_{n} \geq 0 \\
& \sum_{n=0}^{\infty}(n+1)^{-2} \exp \left[(n+1) \int_{0}^{1} x^{n} \log a(x) d x\right] \\
& \leq e^{2-\gamma} \int_{0}^{1} a(x) d x, \quad a(x) \geq 0 .
\end{aligned}
$$

For $\nu=1$, it follows from Corollary 2, where $s=p / q^{\prime}-1$ in (a) and $s=p^{\prime} / q^{\prime}-1$ in (b), and Lemma $1(\mathrm{a})$, that Theorem 3 is also valid when $r=p$, with $1<q \leq p^{\prime}$ in (a) and $1<q \leq p$ in (b), provided that $\left|a_{0}\right|^{p}$ is replaced by $\left|a_{0}\right|^{p} \Gamma\left(p / q^{\prime}\right),\left|\bar{A}_{0}\right|^{p}$ by $\left|A_{0}\right|^{p} \Gamma\left(p^{\prime} / q^{\prime}\right)$, and the weight $(n+1)$ by $n$ for $n \geq 1$. Moreover, the resulting constant $\Gamma(1 / q)^{p}$ is best possible by the same argument given below for (a) when $r=p, q \geq p^{\prime}$. This applies in particular to $\left(\mathrm{B}_{0}\right)$ where $q=p \leq p^{\prime}$ for $1<p \leq 2$ and to $\left(\mathrm{C}_{0}\right)$ where $q=p^{\prime} \leq p$ for $p \geq 2$.

THEOREM 3. For $1<p \leq r<\infty, q>0$, we have

(a)

$$
\left[\int_{0}^{1}|A(x)|^{r}(1-x)^{r / q-1} d x\right]^{1 / r}
$$

$$
\begin{gathered}
<K(p, r, q)\left[\sum_{n=0}^{\infty}\left|a_{n}\right|^{p}(n+1)^{-(p+q-p q) / q}\right]^{1 / p} \\
{\left[\sum_{n=0}^{\infty}\left|A_{n}\right|^{r}(n+1)^{r / q-1}\right]^{1 / r}} \\
<K\left(r^{\prime}, p^{\prime}, q\right)\left[\int_{0}^{1}|a(x)|^{p}(1-x)^{-(p+q-p q) / q} d x\right]^{1 / p}
\end{gathered}
$$


unless the sequence $a_{n}$ is null or $a(x)$ is null a.e., respectively, and where

$$
K(p, r, q)^{r}=[\Gamma(1 / q) \max (1 / \Gamma(1 / p+1 / q), 1)]^{p} \max \left(\Gamma\left(p^{\prime} / q\right), 1\right)^{(r-p) / p^{\prime}} .
$$

Moreover, the constant in (a) is best possible for $r=p, q \geq p^{\prime}$, and in (b) for $r=p$, $q \geq p$.

PROOF. For $p=r$, (a) follows from Corollary 2(a) for $\nu=1 / p+1 / q, s=0$, when applied to the sequence $\left|a_{n}\right|(n+1)^{1-\nu}$, and then the lower estimates for the binomial coefficients in Lemma 2 are substituted in the resulting expression for $A^{(\nu)}(x)$. For $p=r$,

$$
K(p, p, q)=\Gamma(1 / q) \max (1 / \Gamma(1 / p+1 / q), 1) .
$$

For the general case where $r>p$, with $S_{p}$ denoting the normed sum on the righthand side of (a), $c=p^{\prime} / q$, and $\nu=1 / p+1 / q$, we have

$$
\begin{aligned}
|A(x)| & \leq \sum_{n=0}^{\infty}(n+1)^{1-\nu}\left(\left|a_{n}\right|(n+1)^{\nu-1} x^{n}\right) \\
& \leq S_{p}\left[\sum_{n=0}^{\infty}(n+1)^{c-1} x^{n}\right]^{1 / p^{\prime}} \\
& \leq S_{p} \operatorname{Max}(\Gamma(c), 1)^{1 / p^{\prime}}\left[\sum_{n=0}^{\infty}\left(\begin{array}{c}
n+c-1 \\
c-1
\end{array}\right) x^{n}\right]^{1 / p^{\prime}} \\
& =\operatorname{Max}\left(\Gamma\left(p^{\prime} / q\right), 1\right)^{1 / p^{\prime}}(1-x)^{-1 / q} S_{p},
\end{aligned}
$$

by Hoelder's inequality and Lemma 2. Finally, using (a) for $r=p$ and the preceding estimate for $A(x)$, we obtain

$$
\begin{aligned}
\int_{0}^{1}|A(x)|^{r}(1-x)^{r / q-1} d x & \leq \operatorname{Max}\left(\Gamma\left(\frac{p^{\prime}}{q}\right), 1\right)^{(r-p) / p^{\prime}} S_{p}^{r-p} \int_{0}^{1}|A(x)|^{p}(1-x)^{p / q-1} d x \\
& <K(p, p, q)^{p} \operatorname{Max}\left(\Gamma\left(p^{\prime} / q\right), 1\right)^{(r-p) / p^{\prime}} S_{p}^{r}=K(p, r, q)^{r} S_{p}^{r},
\end{aligned}
$$

which proves (a). (b) is derived from (a) by the same duality argument used by Hardy and Littlewood to prove Theorem 4 from Theorem 3 in [4]. In fact, a similar duality argument obtains (a) from (b). Consequently, it suffices to show that the constant is best possible only in (a) when $r=p$ and $q \geq p^{\prime}$ in which case the constant is $\Gamma(1 / q)$. For $0<\varepsilon<1 / q<1$, define the sequence $a_{n}$ so that $a_{0}=\Gamma(1 / q-\varepsilon)$, $a_{n}=n^{\nu-1}$ for $n \geq 1$, where $\nu=1 / q-\varepsilon$. Then we have

$$
\begin{aligned}
S_{p}^{p}-\Gamma(\nu)^{p} & =\sum_{n=1}^{\infty} n^{p(\nu-1)}(n+1)^{-(p+q-p q) / q}=\sum_{n=1}^{\infty}\left(1+\frac{1}{n}\right)^{p(1-\nu)}(n+1)^{-(1+\varepsilon p)} \\
& =\sum_{n=1}^{\infty}(n+1)^{-(1+\varepsilon p)}+\sum_{n=1}^{\infty}\left[\left(1+\frac{1}{n}\right)^{p(1-\nu)}-1\right](n+1)^{-(1+\varepsilon p)} \\
& \leq \frac{1}{\varepsilon p}+p(1-\nu) \sum_{n=1}^{\infty}\left(1+\frac{1}{n}\right)^{p(1-\nu)-1} n^{-(2+\varepsilon p)} \\
& =\frac{1}{\varepsilon p}+O(1)
\end{aligned}
$$


as $\varepsilon \rightarrow 0$, since $p(1-\nu)-1>0$ for $q \geq p^{\prime}$. Therefore, $S_{p}^{p} \leq 1 / \varepsilon p+O(1)$ where $O(1)>0$, and $1 / \varepsilon p \geq S_{p}^{p} /(1+o(1))$ as $\varepsilon \rightarrow 0$ where $o(1)>0$. Finally from Lemma 2 (c) and the last estimate it follows that

$$
\begin{aligned}
& A(x)=\Gamma\left(\frac{1}{q-\varepsilon}\right)+\sum_{n=1}^{\infty} n^{\nu-1} x^{n} \\
& \geq \Gamma\left(\frac{1}{q-\varepsilon}\right) \sum_{n=0}^{\infty}\left(\begin{array}{c}
n+\nu-1 \\
\nu-1
\end{array}\right) x^{n}=\Gamma\left(\frac{1}{q-\varepsilon}\right)(1-x)^{-1 / q-\varepsilon}, \\
& \int_{0}^{1}|A(x)|^{p}(1-x)^{p / q-1} d x>\frac{\Gamma(1 / q)}{\varepsilon p} \geq \frac{\Gamma(1 / q)^{p} S_{p}^{p}}{1+o(1)}, \quad \text { as } \varepsilon \rightarrow 0 .
\end{aligned}
$$

This shows that the constant is best possible and completes the proof.

\section{REFERENCES}

1. D. Borwein, On a scale of Abel-summability methods, Proc. Cambridge Philos. Soc. 53 (1957), 318-322.

2. G. H. Hardy, An inequality for Hausdorff means, J. London Math. Soc. 18 (1943), 45-49.

3. _ Divergent series, Chapter XI, Clarendon Press, Oxford, 1949.

4. G. H. Hardy and J. E. Littlewood, Elementary theorems concerning power series and moment constants, J. für Mat. 157 (1927), 141-158.

5. G. H. Hardy, J. E. Littlewood and G. Pölya, Inequalities, Cambridge Univ. Press, 1967.

6. O. Henriksson, Über die Hausdorffsche Limitierungsverfahren die schwacher sind als das Abelsche, Math. Z. 39 (1935), 501-510.

7. A. Jakimovski, The sequence-to-function analogues to Hausdorff transformations, Bull. Res. Council of Israel Sect. 8F (1960), 135-154.

8. K. Knopp, Über Reihen mit positiven Griedern, J. London Math. Soc. 30 (1930), 13-21.

9. D. Leviatan and L. Lorch, A characterization of totally regular $[J, f(x)]$ transforms, Proc. Amer. Math. Soc. 23 (1969), 315-319.

10. A. Renyi, Summation methods and probability theory, Publ. Math. Inst. Hungar Acad. Sci. 4 (1959), 389-397.

11. D. Widder, The Laplace transform, Princeton Univ. Press, 1946.

Department of Mathematics, DePaul University, Chicago, Illinois 60614 